\title{
PEEC Modeling of Antenna Characteristics
}

\author{
Sofia Sundberg and Jonas Ekman \\ EISLAB \\ Dept. of Computer Science \& Electrical Engineering \\ Luleå University of Technology \\ SE-97187 Luleå \\ Sweden
}

\begin{abstract}
Electromagnetic modeling of antenna characteristics can be performed using different methods. This papers deals with antenna modeling using Partial Element Equivalent Circuit (PEEC) models. Antenna properties including input impedance, radiation diagrams and gain are computed from PEEC model results. The input impedance is computed directly by using the currents and voltages at the feed-point of the antenna. The radiation diagrams are computed in a post-processing step by treating each volume cell as an infinitesimal dipole and using the superposition principle to compute the radiated field. The computed results are compared to analytical results for dipoles. For more complex antenna structures the computed results are compared with measurements and results from other computational methods. Accurate and fast results shows that the method is promising as an antenna system design tool.
\end{abstract}

\section{INTRODUCTION}

There is an increasing number of applications today using wireless communications, from Bluetooth units in a small network around a PC and wireless networks in a building to mobile phones communicating over large distances. Small hand held units today, such as mobile phones or small hand held computers, usually use one or more of these. The typical development of these units today are towards smaller size with longer battery times. This puts new demands on the development of the antennas used. Instead of the traditional monopole-like antennas, smaller integrated antennas are used of more and more complex shape [1], [2]. These antennas usually have several resonance frequencies and strict demand for directivity to prevent radiated power to dissipate in human tissue for example [3]. This calls fore more sophisticated tools for developing antennas, such as [4], [5], [6] since the use of prototypes is expensive and time consuming.

Partial Element Equivalent Circuit (PEEC) models [7], [8], [9] have been shown to be particularly suitable for the solution of electromagnetic compatibility (EMC) and electrical interconnect and package (EIP) problems in combination with SPICE type circuit models since the entire problem is solved in the circuit domain. A key feature of the PEEC approach is that the same circuit models can be constructed for both the time and the frequency domain. The classical PEEC method has evolved towards a complete method in solving more and more complex problems within new areas of application. The combined circuit and electromagnetic solution makes the approach a suitable tool also for antenna design and verification. Since the method offers a direct support for including various circuit elements, which are described by the appropriate MNA stamp [10], the complete antenna system can be analyzed in a straightforward manner.

In this paper, the modeling of antennas by using a PEEC-based electromagnetic solver is shown. Modeling results, input impedance and radiation diagrams, for complex antenna structures are given and modeling possibilities are discussed.

\section{BASIC PEEC THEORY}

This section gives an outline of the nonorthogonal PEEC method as fully detailed in [11]. In this formulation, the objects, conductors and dielectrics, can be both orthogonal and non-orthogonal quadrilateral (surface) and hexahedral (volume) elements. The formulation utilizes a global and a local coordinate system where the global coordinate system uses orthogonal coordinates $x, y, z$. The local coordinates $a, b, c$ are used to separately represent each specific possibly non-orthogonal object and the unit vectors are $\overrightarrow{\hat{a}}, \overrightarrow{\hat{b}}$, and $\overrightarrow{\hat{c}}$, see further [11]. The starting point for the theoretical derivation is the total electric field at a conductor expressed as

$$
\vec{E}^{i}\left(\overrightarrow{r_{g}}, t\right)=\frac{\vec{J}\left(\overrightarrow{r_{g}}, t\right)}{\sigma}+\frac{\partial \vec{A}\left(\overrightarrow{r_{g}}, t\right)}{\partial t}+\nabla \phi\left(\overrightarrow{r_{g}}, t\right),
$$

where $\vec{E}^{i}$ is the incident electric field, $\vec{J}$ is the current density in a conductor, $\vec{A}$ is the magnetic vector poten- 


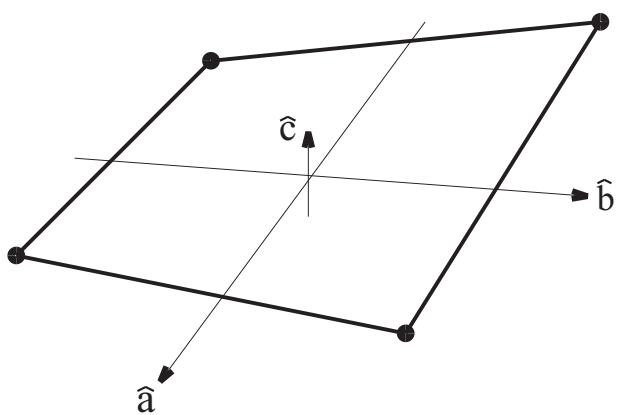

Fig. 1. Nonorthogonal element created by the mesh generator with associated local coordinate system.

tial, $\phi$ is the scalar electric potential, and $\sigma$ the electrical conductivity. The dielectric areas are taken into account as an excess current with the scalar potential using the volumetric equivalence theorem. By using the definitions of the vector potential $\vec{A}$ and the scalar potential $\phi$ we can formulate the integral equation for the electric field at a point $\overrightarrow{r_{g}}$ which is to be located either inside a conductor or inside a dielectric region according to

$$
\begin{aligned}
\vec{E}^{i}\left(\overrightarrow{r_{g}}, t\right) & =\frac{\vec{J}\left(\overrightarrow{r_{g}}, t\right)}{\sigma} \\
& +\mu \int_{v^{\prime}} G\left(\overrightarrow{r_{g}}, \overrightarrow{r_{g}}\right) \frac{\partial \vec{J}\left(\overrightarrow{r_{g}}, t_{d}\right)}{\partial t} d v \prime \\
& +\epsilon_{0}\left(\epsilon_{r}-1\right) \mu \int_{v^{\prime}} G\left(\overrightarrow{r_{g}}, \overrightarrow{r_{g}^{\prime}}\right) \frac{\partial^{2} \vec{E}\left(\overrightarrow{r_{g}}, t_{d}\right)}{\partial t^{2}} \\
& +\frac{\nabla}{\epsilon_{0}} \int_{v^{\prime}} G\left(\overrightarrow{r_{g}}, \overrightarrow{r_{g}}\right) q\left(\overrightarrow{r_{g}}, t_{d}\right) d v \prime
\end{aligned}
$$

The PEEC integral equation solution of Maxwell's equations is based on the total electric field, e.g. (1). An integral or inner product is used to reformulate each term of (2) into the circuit equations. This inner product integration converts each term into the fundamental form $\int \vec{E} \cdot d l=V$ where $V$ is a voltage or potential difference across the circuit element. It can be shown how this transforms the sum of the electric fields in (1) into the Kirchoff Voltage Law (KVL) over a basic PEEC cell [9]. Fig. 2 details the $\left(L_{p}, P, \tau\right)$ PEEC model for the metal patch in Fig. 1 when discretized using four edge nodes (dark full circles). The model in Fig. 2 consists of:

- partial inductances $\left(L_{p}\right)$ which are calculated from the volume cell discretization using a double volume integral.

- coefficients of potentials which are calculated from the surface cell discretization using a double surface integral.
- retarded current controlled current sources, to account for the electric field couplings, given by $I_{p}^{i}=$ $\frac{p_{i j}}{p_{i i}} I_{C}^{j}\left(t-t_{d_{i j}}\right)$ where $t_{d_{i j}}$ is the free space travel time (delay time) between surface cells $i$ and $j$,

- retarded current controlled voltage sources, to account for the magnetic field couplings, given by $V_{L}^{n}=L p_{n m} \frac{\partial I_{m}\left(t-t_{d_{n m}}\right)}{\partial t}$, where $t_{d_{n m}}$ is the free space travel time (delay time) between volume cells $n$ and $m$.

By using the MNA method, the PEEC model circuit elements can be placed in the MNA system matrix during evaluation by the use of correct matrix stamps [10].

\section{Modeling Antenna InPUT IMPEDAnCE}

This section compares results for input impedance values given by PEEC models, analytical formulas, and measurements. For the purpose of modeling antennas, $(L p, P, R, \tau)$ PEEC models have been used for a complete characterization of electric- and magnetic- field couplings. The PEEC-kernel performs the meshing, the calculation of partial elements, and the solution of the time- and/or frequency- domain system. For the antennas analyzed in this paper, the frequency domain solver is used. However, reverting to transient analysis, the antennas can be studied in the time domain as well.

\section{A. Input impedance and reflection coefficient}

Here, the input impedance is given by

$$
Z_{\text {in }}=\frac{V_{i n}}{I_{\text {in }}}
$$

where $V_{i n}$ and $I_{i n}$ is the input voltage and current as given by the PEEC-solver for a specific geometry. This calculation is straightforward due to the circuit based PEEC method.

1) $\frac{\lambda}{2}$ dipole: First we consider a $\frac{\lambda}{2}$ dipole with total length of $20 \mathrm{~cm}$. The theoretical resonance frequency for an $20 \mathrm{~cm}$ finite length dipole is $750 \mathrm{MHz}$ at input impedance close to $73.1 \Omega$. By using a simple PEEC model consisting of 20 cells per arm, the resonance is $752 \mathrm{MHz}$ with an input impedance of $75.5 \Omega$. By using a finer mesh and by successively reducing the cross section of the arms, the results approaches $73.9 \Omega$. For comparison, consider the value $73.5 \Omega$ for the magnitude of the input impedance reported in [11] for a PEECbased EM-solver by using a non-uniform mesh.

2) F-antenna: Continuing to a more complex antenna, the F-antenna in Fig. 3 is modeled. The antenna is tuned for a resonance frequency at $1.5 \mathrm{GHz}$. However, the measurements, performed using a Vector Network 


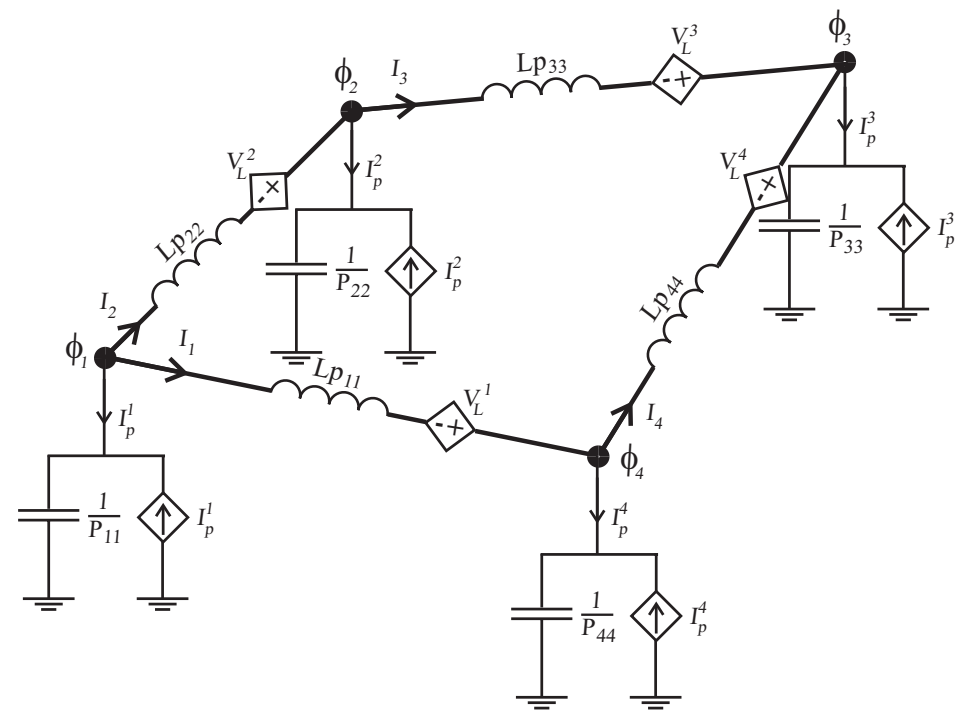

Fig. 2. $\left(L_{p}, P, \tau\right)$ PEEC model for metal patch in Fig. 1 discretized with four edge nodes. Controlled current sources, $I_{p}^{n}$, account for the electric field coupling and controlled voltage sources, $V_{L}^{n}$, account for the magnetic field coupling.

Analyzer, indicate resonance at $1.56 \mathrm{GHz}$ as seen in Fig. 5. To enable comparison of model results with measurements, the rigid coax cable have to be included in the PEEC model (seen as the bar extending to the right in Fig. 3).

With the antenna connected to an coax cable with characteristic impedance $50 \Omega$, the voltage reflection coefficient is defined as

$$
\Gamma=\frac{Z_{\text {in }}-50}{Z_{\text {in }}+50}
$$

from which the reflection coefficient (in $\mathrm{dB}$ ) is calculated as

$$
S 11=-20 \log (\Gamma)
$$

The reflection coefficient for the F-antenna is shown in Figs. 4 (real and imaginary component) and 5 (in $\mathrm{dB}$ ). The comparison with measurements is quite good. It was shown that the level of insertion loss is very sensitive to the cross section of the conductor used for the F-structure of the antenna.

\section{Modeling ANTEnNa RAdiation DiagRam}

\section{A. Theoretical derivation}

When computing the radiation diagram for PEEC models [12], [13], each cell created by the meshing routine is treated as an infinitesimal dipole. The current is assumed to be constant over each cell, and is given by the PEEC-solver, and is used to compute the electrical field for each cell. The complete radiated field is then summarized using the superposition principle.

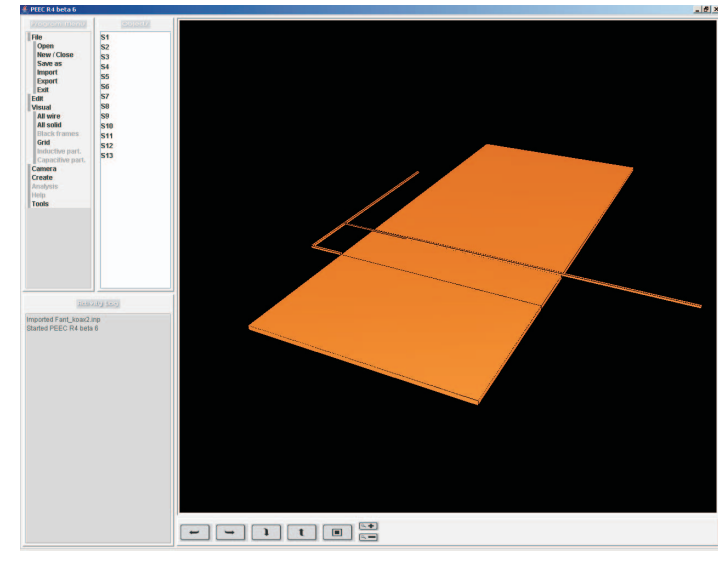

Fig. 3. Geometry for F-antenna with rigid coax cable connected.
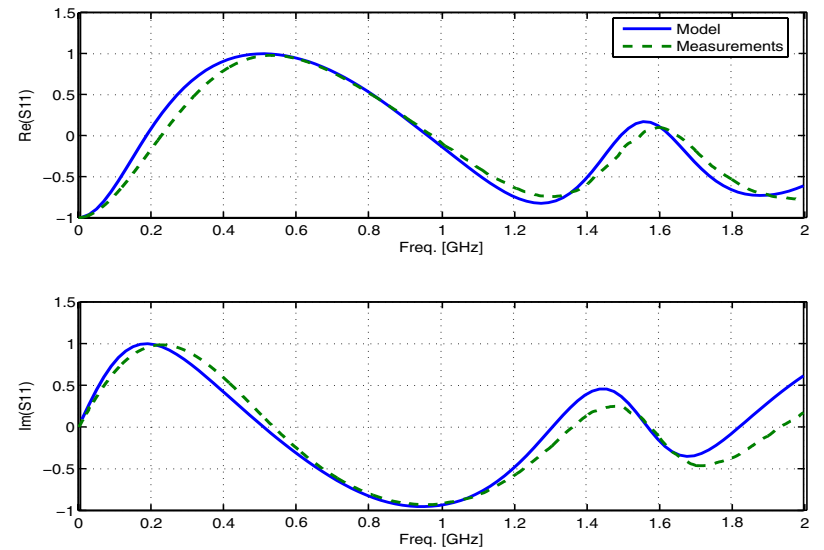

Fig. 4. The imaginary (top) and real (bottom) part of the reflection coefficient for the F-antenna. 


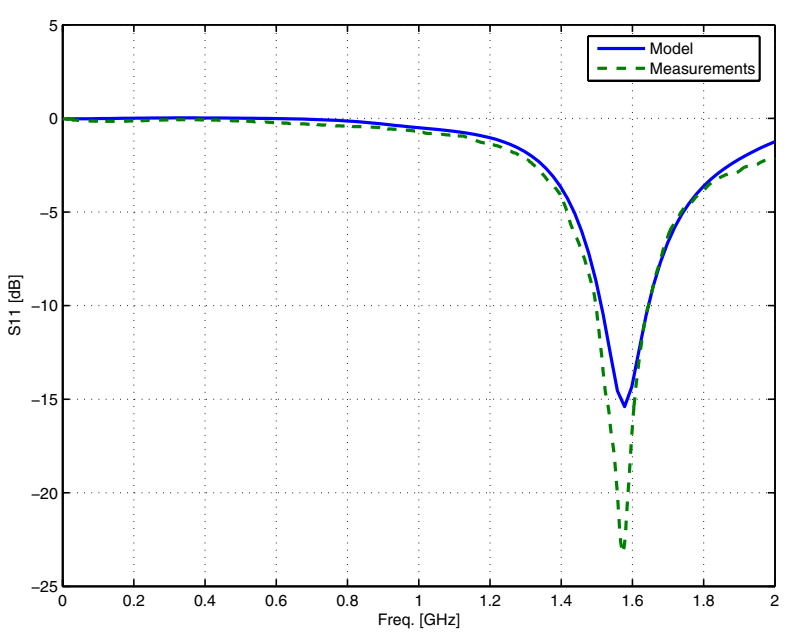

Fig. 5. Reflection coefficient for the F-antenna.

If the volume-cell is meshed in such a manner that it can not be treated as an infinitesimal dipole, a local re-meshing in the post-processing routine of the volume can be performed to fit the dipole assumption in a better way.

To calculate radiated field form an infinitesimal dipole of length $l$, the starting point is the magnetic vector potential defined [14] as

$$
\mathbf{A}(x, y, z)=\frac{\mu}{4 \pi} \int_{C} \mathbf{I}\left(x^{\prime}, y^{\prime}, z^{\prime}\right) \frac{e^{-\jmath k R}}{R} d l^{\prime}
$$

where $(x, y, z)$ and $\left(x^{\prime}, y^{\prime}, z^{\prime}\right)$ are the observation and source points respectively, and $\mathbf{I}$ the constant current in the cell. Further, $R$ is the distance from the source to the observation point.

By placing the infinitesimal dipole along the z-axis centered at origo in an rectangular coordinate system, the current is $\mathbf{I}=\hat{\mathbf{z}} I_{z}$ and

$$
\mathbf{A}(x, y, z)=\hat{\mathbf{z}} \frac{\mu I_{z}}{4 \pi R} \int_{-\frac{l_{z}}{2}}^{\frac{l_{z}}{2}} d z^{\prime}=\hat{\mathbf{z}} \frac{\mu I_{z} l_{z}}{4 \pi R} e^{-\jmath k R}
$$

where $R=\sqrt{x^{2}+y^{2}+z^{2}}=$ constant and $d z^{\prime}=d l^{\prime}$. A transformation to the more suitable spherical coordinate system results in

$$
\begin{aligned}
& A_{r}=A_{z} \cos \theta=\frac{\mu I_{z} l_{z}}{4 \pi R} e^{-\jmath k R} \cos \theta \\
& A_{\theta}=-A_{z} \sin \theta=-\frac{\mu I_{z} l_{z}}{4 \pi R} e^{-\jmath k R} \sin \theta \\
& A_{\phi}=0 .
\end{aligned}
$$

To calculate the radiated electric field from the in- finitesimal dipole, the magnetic field intensity defined as

$$
\begin{aligned}
\mathbf{H}_{A}= & \frac{1}{\mu}\left\{\hat{\mathbf{r}} \frac{1}{r \sin \theta}\left[\frac{\partial}{\partial \theta}\left(A_{\phi} \sin \theta\right)-\frac{\partial A_{\theta}}{\partial A_{\phi}}\right]\right. \\
& +\frac{\hat{\theta}}{\mu R}\left[\frac{\partial A_{r}}{\partial A_{\phi}} \frac{1}{\sin \theta}-\frac{\partial}{\partial r\left(R A_{\phi}\right)}\right] \\
& \left.+\frac{1}{\mu} \frac{\phi}{R}\left[\frac{\partial}{\partial r}\left(R A_{\theta}\right)-\frac{\partial A_{r}}{\partial \theta}\right]\right\}
\end{aligned}
$$

is used. According to the spherical symmetry of the problem and since there are no $\phi$ variations along the $\mathrm{z}$-axis, the following is given

$$
\mathbf{H}_{A}=\hat{\phi} \frac{k \jmath I_{z} l_{z} \sin \theta}{4 \pi R}\left(1+\frac{1}{\jmath k R}\right) e^{-\jmath k R} .
$$

Equating Maxwell's equations

$$
\nabla \times \mathbf{H}_{A}=\mathbf{J}+\jmath \omega \epsilon \mathbf{E}_{A}
$$

and excluding the current density $\mathbf{J}=0$, the electric field can be written as

$$
\begin{aligned}
& E_{r}=\eta \frac{I_{z} l_{z} \cos \theta}{2 \pi R^{2}}\left[1+\frac{1}{\jmath k R}\right] e^{-\jmath k R} \\
& E_{\theta}=\jmath \eta \frac{k I_{z} l_{z} \sin \theta}{4 \pi R}\left[1+\frac{1}{\jmath k R} \frac{1}{k R^{2}}\right] e^{-\jmath k R} \\
& E_{\phi}=0
\end{aligned}
$$

where $\eta=\frac{E_{\theta}}{H_{\phi}}$ is the intrinsic impedance $(=120 \pi \mathrm{ohms}$ for free space). In the far-field region, $k R \gg 1$, gives

$$
E_{\theta}=\jmath \eta \frac{k I_{z} l_{z} e^{-\jmath k R}}{4 \pi R} \sin \theta
$$

and

$$
E_{r} \simeq E_{\phi}=0
$$

which is the far-field solution for an infinitesimal dipole along the $z$-axis. The same procedure can be used to solve the electric field for an infinitesimal dipole along the $x$-axis and along the $y$-axis. In both cases, $E_{r} \simeq$ 0 . The electric field for an infinitesimal dipole in origo along the $x$-axis is

$$
\begin{aligned}
& E_{\theta} \simeq-\jmath \eta \frac{k I_{x} l_{x} e^{-\jmath k R}}{4 \pi R} \cos \theta \cos \phi, \\
& E_{\phi} \simeq-\jmath \eta \frac{k I_{x} l_{x} e^{-\jmath k R}}{4 \pi R} \sin \phi .
\end{aligned}
$$

where $I_{x}$ is the constant current and $l_{x}$ is the length of the dipole in the current direction. Finally, for an infinitesimal dipole with the currents in the $y$-direction the electric far field can be calculated using

$$
\begin{aligned}
& E_{\theta} \simeq-\jmath \eta \frac{k I_{y} l_{y} e^{-\jmath k R}}{4 \pi R} \cos \theta \sin \phi, \\
& E_{\phi} \simeq-\jmath \eta \frac{k I_{y} l_{y} e^{-\jmath k R}}{4 \pi R} \cos \phi .
\end{aligned}
$$


When computing the far-field for PEEC model current/volume cells the coordinates for each field point and current cell are in the Cartesian coordinate system. The expressions for $\sin \theta, \cos \theta, \sin \phi$ and $\cos \phi$ is computed as $\sin \theta=\frac{r_{p}}{r}, \cos \theta=\frac{\Delta z}{r}, \sin \phi=\frac{\Delta y}{r_{p}}$, and $\cos \phi=\frac{\Delta x}{r_{p}}$, where $\Delta x, \Delta y$ and $\Delta y$ is the distance between the field point and center point of the current cell in the $x$ - $y$ - and $z$-direction respectively, $r=\sqrt{\Delta x^{2}+\Delta y^{2}+\Delta z^{2}}$ is the distance between the field point and the center point of the current cell and $r_{p}=\sqrt{\Delta x^{2}+\Delta y^{2}}$ is the distance projected down on the $x y$-plane.

The entire field $\mathbf{E}^{\mathbf{p}}$ at field point $p$ is summarized as

$$
\mathbf{E}^{\mathbf{p}}=\sum_{i=1}^{n} \overrightarrow{E^{i}}
$$

where $n$ is the number of volume current cells and $\vec{E}^{i}$ is the contribution of the field from volume cell $i$.

\section{B. Antenna gain}

A more general way of describing the performance of an antenna is by computing the gain of the antenna. The gain is computed as

$$
G=4 \pi \frac{U(\theta, \phi)}{P_{\text {in }}}
$$

where $P_{i n}$ is total input power to the antenna given by $P_{\text {in }}=Z_{\text {in }} I_{\text {in }}^{2}$ and $U(\theta, \phi)$ is the radiation intensity. The radiation intensity is related to the far-field as

$$
U(\theta, \phi) \simeq \frac{r^{2}}{\eta}\left[\left|E_{\theta}^{2}+E_{\phi}^{2}\right|\right]
$$

when using RMS values and $r$ is the distance to the center point of the antenna.

\section{Results}

1) $\frac{\lambda}{2}$ dipole: To allow for comparison to analytical results, the electric field for the previously considered $20 \mathrm{~cm}$ dipole has been modeled. The model uses 20 cells per arm and is excited using a current source in the gap between the dipole arms at $750 \mathrm{MHz}$. From the comparison in Fig. 6 and 7 it is clear that the approach is suitable for calculating radiated electric far-fields and the maximal error is less than $1 \%$ in the $x y$-plane. The error in the $y z$-plane is somewhat larger, less than $5 \%$, for the extreme angels $0^{\circ}$ and $180^{\circ}$ where the $E_{\theta}$ component is contributing to the total field.

The results can be improved by, for example, improving the meshing of the dipole arms.

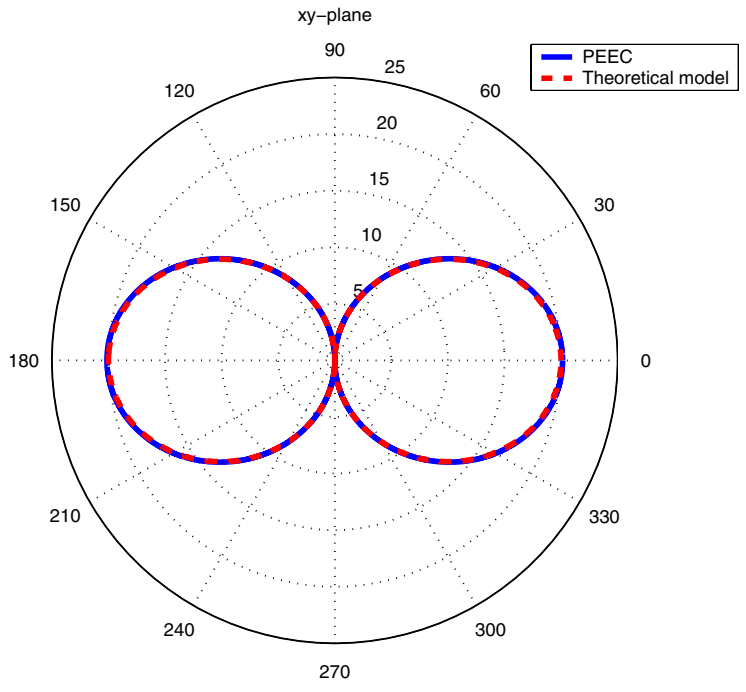

Fig. 6. E-field [V/m] at $3 \mathrm{~m}$. for long dipole in $x$-direction by PEEC solver compared to the theoretical values for the $x y$-plane.

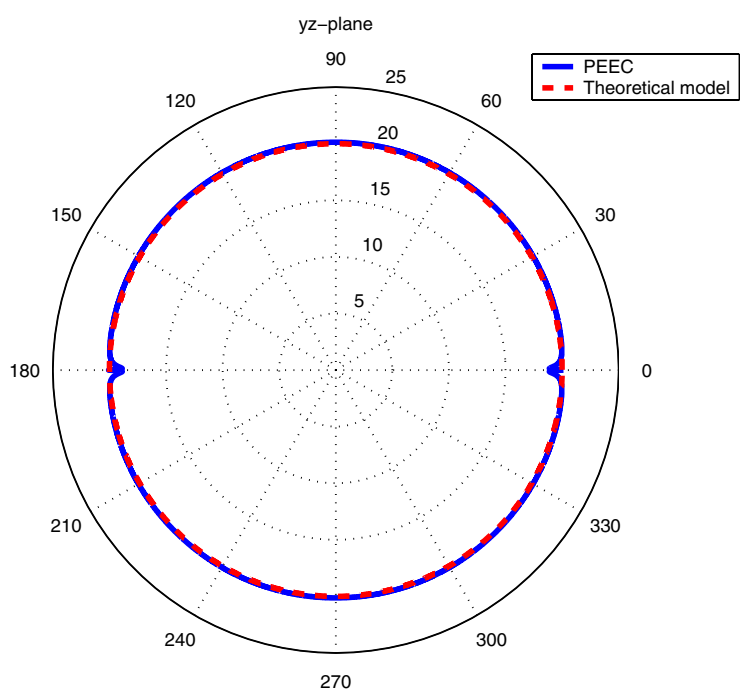

Fig. 7. E-field [V/m] at $3 \mathrm{~m}$. for long dipole in $x$-direction by PEEC solver compared to the theoretical value for the $y z$-plane.

2) F-antenna: The gain from the F-antenna was also computed by the PEEC-solver. To allow comparison with another method, the same problem was modeled in CST Microwave Studio [15]. The results are shown in Fig. 8 and 9 for the gain in the $x y$ - and $y z$-plane respectively and compare well. As seen in Fig. 8 the $x y$-plane is the most difficult direction to model. This is due to the fact that the antenna is oriented in the $x y$-plane and the $\Delta z$ variable in the angle computations will be close to 0 which will affect the numerical errors in the angle computations. A possible solution is to identify this based on the discretization, there are no volume cells in 


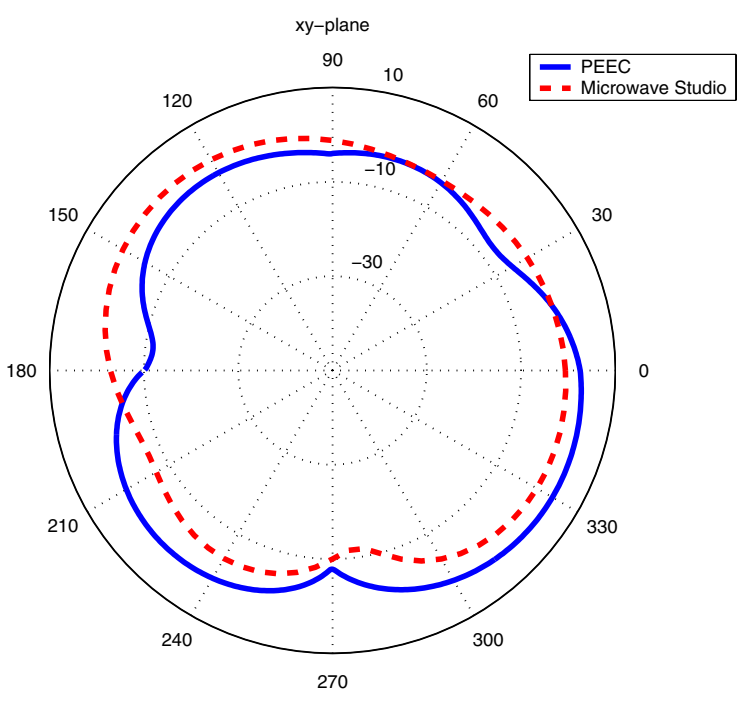

Fig. 8. Gain in $\mathrm{dB}$ in the $\mathrm{xy}$-plane from PEEC solver and CST Microwave Studio.

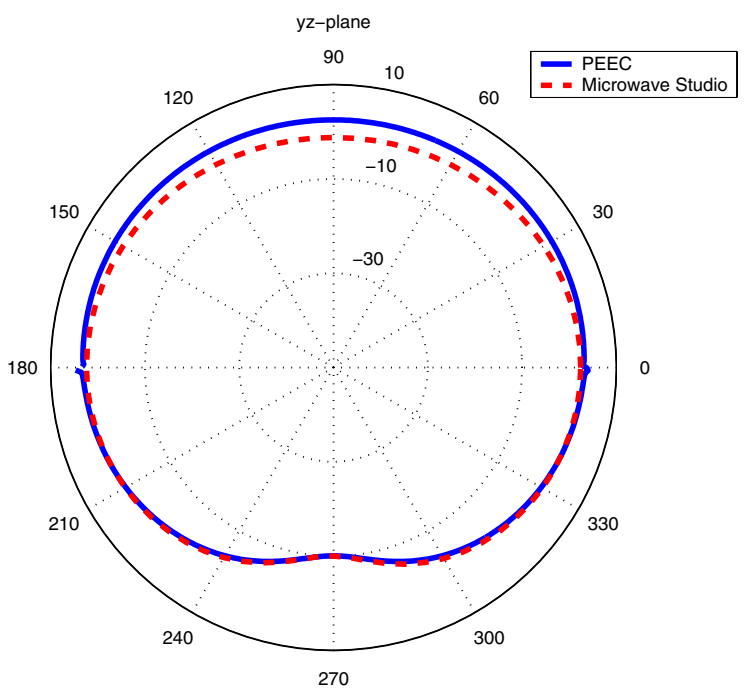

Fig. 9. Gain in $\mathrm{dB}$ in the yz-plane from PEEC solver and CST Microwave Studio.

the $z$-direction for the F-antennna, and automatically set the $\Delta$-terms to 0 if needed.

\section{TIME COMPleXity}

The computation of radiated fields from PEEC model results does not add a considerable amount of time to the overall solution. Consider that the time complexity of the frequency domain PEEC solver without acceleration techniques is $O\left(n^{3}\right)$ where $n$ is the number of unknown currents and voltages. The time complexity of the postprocessing (field computation) is $O\left(m_{f} n_{v}\right)$ where $m_{f}$ is the number of field points and $n_{v}$ is the number of volume cell currents $\left(n_{v}<<n\right)$.

\section{CONCLUSiOnS}

This paper shows the application of PEEC models for antenna analysis and the computation of electric fields and gain in a post-processing fashion. It is clear that PEEC models are suitable for antenna analysis and results shown here compares well with both measured values and results from other EM-solvers. Further work involves modeling of more complicated antennas and study on radiation efficiency.

\section{REFERENCES}

[1] Z.N. Low, J.H. Cheong, and C.L. Law, "Low-Cost PCB Antenna for UWB Applications".IEEE Antennas and Wireless Propagation Letters, 4:237-239, 2005.

[2] C. A. Macon, L. C. Kempel, S. W. Schneider, and K. D. Trott, "Modeling Conformal Antennas on Metallic Prolate Spheroid Surfaces Using a Hybride Finite Element Method", IEEE Trans. on Antennas and Propagation, 52(3):750-758, March 2004.

[3] Dong-Uk Sim and Seong-Ook Park, "A Triple-Band Internal Antenna: Design and Performance in Presence of the Handset Case, Battery, and Human Head", IEEE Trans. on EMC, 47(3):658666, August 2005.

[4] X. Xiaojing, Z. Qi, and L. Yan, "Input Analysis of Spherical Conformal Antennas by Means of Conformal Transformation", in: Proc. IEEE Int. Symp. Antennas and Propagation Society 395-398, June 2004.

[5] T. F. Eibert, K. Sertel, and J. L. Volakis, "Hybride finite element modeling of conformal antenna and array structures utilizing fast integral methods.", Int Journal of Numerical Modeling: Electronic Network, Devices and Fields 13(2-3), March-June 2000

[6] A. Alú, F. Bilotti, and L. Vegni, "Method of Lines Numerical Analysis of Conformal Antennas.", IEEE Trans. on Antennas and Propagation, 52(6):1530-1540, June 2004.

[7] A. E. Ruehli, "Inductance calculations in a complex integrated circuit environment", IBM Journal of Research and Development, 16(5):470-481, September 1972.

[8] A. E. Ruehli and P. A. Brennan, "Efficient capacitance calculations for three-dimensional multiconductor systems", IEEE Trans. on Microwave Theory and Techniques, 21(2):76-82, February 1973.

[9] A. E. Ruehli, "Equivalent circuit models for three-dimensional multiconductor systems", IEEE Trans. on Microwave Theory and Techniques, 22(3):216-221, March 1974.

[10] C. Ho, A. Ruehli, and P. Brennan, "The modified nodal approach to network analysis", IEEE Trans. on Circuits and Systems, pp. 504-509, June 1975.

[11] A. E. Ruehli et al., "Nonorthogonal PEEC formulation for time- and frequency-domain modeling". IEEE Trans. on EMC, 45(2):167-176, May 2003.

[12] H. Heeb, A. Ruehli, J. Janak, and S. Daijavad, "Simulating Electromagnetic Radiation of Printed Circuit Boards". in Proc. ICCAD, pp. 392-395, November 1990.

[13] H.Heeb and A. Ruehli, "Retarded Models for PC Board Interconnect - or How the Speed of Light Affects Your SPICE Circuit Simulation". in Proc. ICCAD, pp. 70-73, November 1991.

[14] C. A. Balanis, Antenna Theory: Analysis and Design. John Wiley Sons, Inc., 2005.

[15] Computer Simulation Technology. [Online]. Available: http://www.cst.com/ 